BNL-112532-2016-JA

\title{
Engineered Pinning Landscapes for Enhanced 2G Coil Wire
}

\author{
Martin W. Rupich, Srivatsan Sathyamurthy, Steven Fleshler, \\ Qiang Li, Vyacheslav Solovyov, Toshinori Ozaki, Ulrich Welp, \\ Wai-Kwong Kwok, Maxime Leroux, Alexei E. Koshelev, Dean J. Miller, \\ Karen Kihlstrom, Leonardo Civale, Serena Eley, and Asghar Kayani
}

Submitted to IEEE Transactions on Applied Superconductivity

April 2016

Condensed Matter Physics and Material Science Department

Brookhaven National Laboratory

\section{U.S. Department of Energy USDOE Office of Science (SC), Basic Energy Sciences (BES) (SC-22)}

Notice: This manuscript has been authored by employees of Brookhaven Science Associates, LLC under Contract No. DE-SC0012704 with the U.S. Department of Energy. The publisher by accepting the manuscript for publication acknowledges that the United States Government retains a non-exclusive, paid-up, irrevocable, world-wide license to publish or reproduce the published form of this manuscript, or allow others to do so, for United States Government purposes. 


\section{DISCLAIMER}

This report was prepared as an account of work sponsored by an agency of the United States Government. Neither the United States Government nor any agency thereof, nor any of their employees, nor any of their contractors, subcontractors, or their employees, makes any warranty, express or implied, or assumes any legal liability or responsibility for the accuracy, completeness, or any third party's use or the results of such use of any information, apparatus, product, or process disclosed, or represents that its use would not infringe privately owned rights. Reference herein to any specific commercial product, process, or service by trade name, trademark, manufacturer, or otherwise, does not necessarily constitute or imply its endorsement, recommendation, or favoring by the United States Government or any agency thereof or its contractors or subcontractors. The views and opinions of authors expressed herein do not necessarily state or reflect those of the United States Government or any agency thereof. 


\title{
Engineered Pinning Landscapes for Enhanced 2G Coil Wire
}

\author{
Martin W. Rupich, Srivatsan Sathyamurthy, Steven Fleshler, Qiang Li, Vyacheslav Solovyov, Toshinori Ozaki, \\ Ulrich Welp, Wai-Kwong Kwok, Maxime Leroux, Alexei E. Koshelev, Dean J. Miller, Karen Kihlstrom, Leonardo \\ Civale, Serena Eley, and Asghar Kayani
}

\begin{abstract}
We demonstrate a 2-fold increase of the in-field critical current of AMSC's standard 2G coil wire by irradiation with $18 \mathrm{MeV}$ Au ions. The optimum pinning enhancement is achieved with a dose of $6 \times 10^{11}$ Au ions $/ \mathrm{cm}^{2}$. Although the $77 \mathrm{~K}$, self-field critical current is reduced by about $35 \%$, the in-field critical current $(\mathrm{H} / / \mathrm{c})$ shows a significant enhancement between 4 - 50K in fields $>1 \mathrm{~T}$. The process was used for the roll-to-roll irradiation of AMSC's standard $46 \mathrm{~mm}$ wide production coated conductor strips which were further processed in to standard copper laminated coil wire. The long length wires show the same enhancement as attained with short static irradiated samples. The roll-to-roll irradiation process can be incorporated in the standard 2G wire manufacturing with no modifications to the current process. The enhanced performance of the wire will benefit rotating machine and magnet applications.
\end{abstract}

Index Terms - Critical current density, flux pinning, hightemperature superconductors, irradiation, roll-to-roll processing.

This work was supported in part by the Advanced Research Projects Agency - Energy, and the Center for Emergent Superconductivity, an Energy Frontier Research Center funded by the US Department of Energy, Office of Science, Office of Basic Energy Sciences.

M. W. Rupich is with American Superconductor Corporation, Devens, MA 01434 (marty.rupich@amsc.com)

S. Sathyamurthy is with American Superconductor Corporation, Devens, MA 01434 (srivatsan.sathymurthy@amsc.com)

S. Fleshler is with American Superconductor Corporation, Devens, MA 01434 (steve.fleshler@amsc.com)

Q. Li is with Brookhaven National Laboratory, Upton, NY 11973 (liqiang@bnl.gov)

V. Solovyov is with Brookhaven National Laboratory, Upton, NY 11973 (solov@bnl.gov)

T. Ozaki is with Brookhaven National Laboratory, Upton, NY 11973 (ozaki@bnl.gov)

U. Welp is with Argonne National Laboratory, Argonne, IL 60439 (welp@anl.com)

W-K. Kwok is with Argonne National Laboratory, Argonne, IL 60439 (kwok@anl.com)

M. Leroux is with Argonne National Laboratory, Argonne, IL 60439 (mleroux@anl.com)

A. E. Koshelev is with Argonne National Laboratory, Argonne, IL 60439 (koshelev@anl.com)

D. J. Miller is with Argonne National Laboratory, Argonne, IL 60439 (miller@nal.com)

K. Kihlstrom is with Argonne National Laboratory, Argonne, IL 60439 (kihlstrom@anl.com)

L. Civale is with Los Alamos National Laboratory, Los Alamos, NM 87545 (lcivale@lanl.gov)

S. Eley is with Los Alamos National Laboratory, Los Alamos, NM 87545 (seley@lanl.gov)

A. Kayani is with Western Michigan University, Kalamazoo MI 49008 (Asghar.Kayani@wmich.edu)

\section{INTRODUCTION}

$\mathrm{S}_{\text {superconducting wires, based on the }}^{\text {ECOND }(\mathrm{RE}) \mathrm{Ba}_{2} \mathrm{Cu}_{3} \mathrm{O}_{7-\delta}(\mathrm{RE}}$ $=$ rare earth elements) materials, are being developed for a number of electric power applications and are expected to provide significant benefits to a range of applications [1]-[3] . The major anticipated applications include cables that operate at high temperatures $(65-77 \mathrm{~K})$ and relatively low fields and rotating machines that operate at a temperature of $30-50 \mathrm{~K}$ range in fields of $1-4 \mathrm{~T}$. Although the performance of the production length $2 \mathrm{G}$ wire is nearing the level required for commercial cable applications, the severe drop in critical current density (Jc) in magnetic fields limits it use in commercial rotating machines. Thus increasing the current carried by second generation (2G) high temperature superconducting (HTS) wire in the presence of high magnetic fields is critical for the commercialization of HTS based rotating machine applications (generators for off-shore wind turbines, hydro power and utility applications; compact, lightweight marine propulsion; etc.) and various HTS magnet applications.

Research over the past few years has shown that pinning landscapes based on mixed defect structures are effective in improving the 2G HTS wire performance [4]. Although mixed pinning structures based on self-assembled $\mathrm{BaZrO}_{3}$ columns and nano-particle defects have been demonstrated in short samples of vapor phase grown (RE)BCO films, it has been difficult to uniformly incorporate these highly engineered defect structures into production length conductors, resulting in significant variation in the critical current $\left(I_{c}\right)$ along the wire length at the operating conditions. This is critically important for commercial $2 \mathrm{G}$ wires since performance of the entire long length wire is limited by the section with the lowest performance. Additionally, these self-assembled columnar structures are not viable options for solution-grown REBCO films using metal organic deposition (MOD). Thus we have been exploring alternative routes to fabricate precisely engineered defect structures into production length MODbased REBCO films.

Nano-particles of various compositions can be introduced into the MOD-based films and have provided significant improvements in the pinning microstructure. The current stateof-the-art MOD REBCO-based 2G wire incorporates a mixed pinning landscape consisting of a dispersion of nano-particles of various sizes, stacking faults and dislocations. Although the development of this pinning microstructure has resulted in a near doubling of in-field $J_{c}$ in the $20-50 \mathrm{~K}$ range, the $I_{c}$ level of actual wires is at least a factor of 2 below that required 
for commercial rotating machine applications. Research over the past year has shown that the addition of point defects, produced by proton irradiation, significantly improves the pinning in the state-of-the-art MOD-based REBCO films, with the critical current $\left(I_{c}\right)$ more than doubling at $\sim 30 \mathrm{~K}$ in magnetic fields $>2.5 \mathrm{~T}$ [5]. This earlier work used $4 \mathrm{MeV}$ protons that produced additional $\sim 5$ nanometer size defects in the existing microstructure, but required long exposure times. Similar performance enhancements have been achieved with much shorter exposure times with $4-14 \mathrm{MeV}$ oxygen ions [6]. These results demonstrate these nanometer sized defects are highly effective at pinning vortices in high fields in MODbased REBCO films. However, they are only relevant to high volume production of $2 \mathrm{G}$ wire if the ion species and energies can be optimized to allow very short exposure times.

In this presentation, we will describe the pinning enhancements achieved in AMSC's production wire using Au ions with an energy around $16 \mathrm{MeV}$. The $J_{c}$ enhancement was similar to that achieved with the proton or $\mathrm{O}$ irradiation. In contrast to proton or $\mathrm{O}$ irradiation, the dosages required to achieve the equivalent pinning enhancement were significantly lower, making roll-to-roll irradiation of production length conductors viable using readily available electrostatic generators.

\section{IRRADIATION PROCESS}

\section{A. Experimental Details}

The coated conductor samples used in this study were taken from AMSC's 2G HTS wire production line. The samples consisted of a $75 \mu \mathrm{m}$ thick $\mathrm{Ni}(5 \mathrm{at} \%) \mathrm{W}$ RABiTs ${ }^{\mathrm{TM}}$ substrate with a buffer stack consisting of $75 \mathrm{~nm}$ each of $\mathrm{Y}_{2} \mathrm{O}_{3}$, YSZ and $\mathrm{CeO}_{2}$ deposited by reactive sputtering and a $1.2 \mu \mathrm{m}$ thick YBCO layer, doped with $\mathrm{Dy}_{2} \mathrm{O}_{3}$, deposited by an MOD process. Approximately $1 \mu \mathrm{m}$ of Ag was deposited on both sides of the tape. The samples were processed as AMSC's coil wire formulation which has a $77 \mathrm{~K}$, self-field $I_{c}$ of around $385 \mathrm{~A} / \mathrm{cm}-\mathrm{w}$ and enhanced pinning at lower temperatures. Samples were cut from the $46 \mathrm{~mm}$ wide strip after the oxygenation step in AMSC's RABiTS/MOD-YBCO manufacturing process [7].

The ion irradiations were carried out at Brookhaven National Laboratory using the tandem Van de Graaff Facility which consists of two $15 \mathrm{MeV}$ electrostatic accelerators. The ion beam consisted of Au ions with either a $5+$ or $6+$ charge with an energy of $16-18 \mathrm{MeV}$. The Au ions were generated from a sputtering source and the ion beam had a particle current of approximately $120 \mathrm{nA}$ at the sample. The samples were irradiated with the ion beam oriented along the crystallographic $c$-axis of the YBCO.

Short samples were irradiated by moving the sample under a static beam for a set time to achieve the targeted exposure. $46 \mathrm{~mm}$ wide, long length production strips were irradiated in a roll-to-roll configuration by rastering the ion beam across the width of the moving strip at a frequency of about $40 \mathrm{~Hz}$. The line speed of the tape was varied to achieve the targeted exposure.
Critical currents were measured using a 4-point transport technique with $1 \mu \mathrm{V} / \mathrm{cm}$ voltage criteria.

The normalized relaxation rates (flux creep rate) were measured in a commercial SQUID magnetometer on small square samples [8]. The rates were obtained from the time decay of $\mathrm{M}_{\text {irr }}$ over a period of 1 hour at fixed temperature and field, after the field was appropriately cycled to create the initial fully penetrated critical state.

\section{B. Short Sample Irradiation}

Some of the earlier irradiation studies were carried out on YBCO films with no Ag coating or at most an extremely thin layer [9]. However, studies have shown that the post annealing of the samples at temperatures above $300^{\circ} \mathrm{C}$ essentially removes the defects from the YBCO film. Thus if the irradiation technique is to be considered for a manufacturing process, it must be carried out after the Ag layer deposition and oxygenation steps, since these processes generally require temperatures in excess of $300^{\circ} \mathrm{C}$. Thus the energy of the ions must be high enough to fully penetrate the Ag layer and still have sufficient energy to cause displacements uniformly though the entire thickness of the YBCO layer.

SRIM-TRIM calculations [10] for $\mathrm{Au}$ ions indicate that $\mathrm{Au}$ ions with an energy of around $16-18 \mathrm{MeV}$ are required to uniformly irradiate a $1.2 \mu \mathrm{m}$ thick YBCO layer through a 1 $\mu \mathrm{m}$ thick Ag layer. If a thicker Ag layer is used, a higher energy for the $\mathrm{Au}$ ions is required.

Previous irradiation studies of AMSC coated conductor tapes using either $\mathrm{H}$ or $\mathrm{O}$ ions showed that a dosage of $2 \times 10^{17}$ $\mathrm{H} / \mathrm{cm}^{2}$ and $1 \times 10^{13} \mathrm{O} / \mathrm{cm}^{2}$ was required to achieve the maximum pinning enhancement in moderate fields at around $30 \mathrm{~K}$. However, SRIM calculations indicate that Au ions will produce significantly more displacements per collision than either $\mathrm{O}$ or $\mathrm{H}$. Thus the required dosage should be significantly less.

Our initial studies were carried out on $1 \times 2 \mathrm{~cm}$ coupons using $\mathrm{Au}^{5+}$ ions with an energy of $18 \mathrm{MeV}$. Samples were exposed to the ion beam for various times to achieve a dose of $1 \times 10^{11} \mathrm{Au} / \mathrm{cm}^{2}$ to $2 \times 10^{12} \mathrm{Au} / \mathrm{cm}^{2}$. Fig. 1 , which plots $I_{c}$ as a function of magnetic field $(\mathrm{H} / / \mathrm{c})$ at $27 \mathrm{~K}$ for the different doses of $\mathrm{Au}$ ions, shows that a maximum enhancement is attained around $6 \times 10^{11} \mathrm{Au} / \mathrm{cm}^{2}$. Fig. 2 shows that the $I_{c}$ enhancement for the optimum dose relative to the unirradiated sample at 30 and $77 \mathrm{~K}$. At low fields and high temperature, the $I_{c}$ of the irradiated sample is reduced; however, at temperatures and fields relevant to rotating machine applications the $I_{c}$ enhancement is over 2-fold and increases with increasing field up to at least $6 \mathrm{~T}$.

In order to establish whether similar enhancements can be achieved on a moving sample, the coupon sample holder was modified so that a $1 \mathrm{~m} \times 1 \mathrm{~cm}$ tape could be transported through the static ion beam. The tape speed was adjusted to achieve the optimal dose of $6 \times 10^{11} \mathrm{Au} / \mathrm{cm}^{2}$. Before the experiment, the beam was shaped and the $x y$ profile determined to establish the irradiation area and beam intensity. Fig. 2 also shows that the $I_{c}$ enhancement measured on the moving sample was nearly identical to that obtained on the 
short static samples.

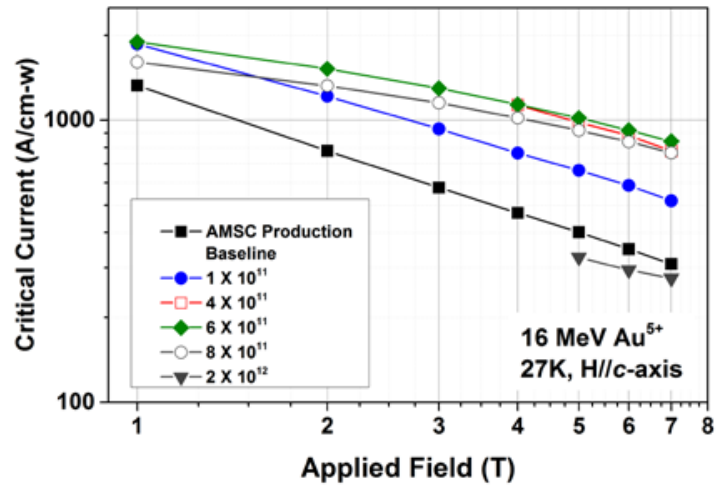

Fig. 1. $I_{c}$ as a function of magnetic field $(\mathrm{H} / / \mathrm{c})$ at $27 \mathrm{~K}$ for the different doses of Au ions.

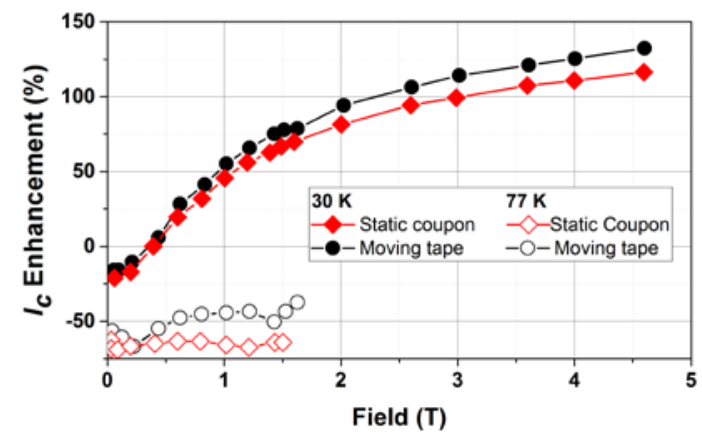

Fig.2. $I_{c}$ enhancement at 30 and $77 \mathrm{~K}$ (relative to an unirradiated control sample) of a stationary short sample and a moving 1 meter tape after irradiation with $18 \mathrm{MeV}$ Au to a dose of $6 \times 10^{11} \mathrm{Au} / \mathrm{cm}^{2}$

Figure 3 shows the normalized relaxation rate, $\mathrm{S}(\mathrm{T})$, for a sample irradiated with $18 \mathrm{MeV}$ Au ions to a dose of $6 \times 10^{11}$ ions $/ \mathrm{cm}^{2}$ and two unirradiated samples, for fields of (a) $0.3 \mathrm{~T}$ and (b) $1 \mathrm{~T}$ oriented parallel to the $c$-axis. Below $\sim 20 \mathrm{~K}$, the creep rates for the irradiated and pristine samples are similar, suggesting that vortex dynamics is dominated by pre-existing pinning. The increasing $S(T)$ can be understood within the framework of the classic Anderson-Kim model [11] that predicts an approximately linear $\mathrm{S}(\mathrm{T})$-relation of the form $\mathrm{S}=$ $k_{B} T / U_{0}$, where $U_{0}$ is the dominant vortex pinning potential. $\mathrm{S}(\mathrm{T})$ does not extrapolate to zero with either sample, indicative of a contribution due to quantum creep at low temperatures [12]. Many previous studies show similar S(T) in this temperature range for YBCO samples with vastly different pinning properties [13,14,15].

At temperatures above $20 \mathrm{~K}$, all samples show a clear departure from the almost linear $\mathrm{S}(\mathrm{T})$ dependence suggesting a fundamental change in the vortex dynamics with a crossover in vortex pinning behavior from mostly strong single-vortex pinning at low temperatures to mostly collective pinning at high temperatures $[12,13,14]$. In the pristine samples, $S(T)$ is strongly non-monotonic and exhibits a minimum around $40 \mathrm{~K}$ with values of $\mathrm{S}<0.02$ that are remarkably low for YBCO at these temperatures. A similar minimum appears in other YBCO films with pinning dominated by large nanoparticles and is likely related to the large pinning energy of the individual nanoparticles Error! Bookmark not defined.[15].
The creep rate above $20 \mathrm{~K}$ is much higher for the irradiated sample, consistent with a scenario where pinning is dominated by smaller defects resulting in the well-known plateau in S(T) [12]-[14].

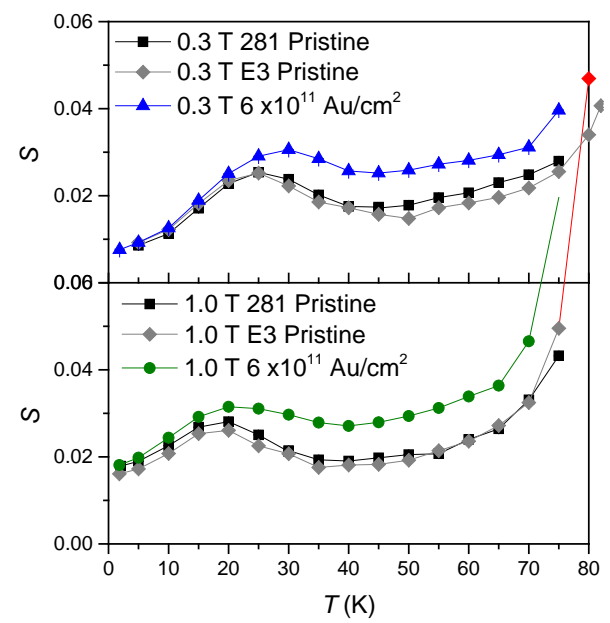

Fig. 3: Flux creep rate as a function of temperature for an Au irradiated sample at $0.3 \mathrm{~T}$ (upper figure) and $1.0 \mathrm{~T}$ (lower figure). $\mathrm{S}(\mathrm{T})$ for unirradiated controls are included for comparison.

\section{Wide Tape Roll-to-Roll Irradiation}

The irradiation of a $46 \mathrm{~mm}$ wide production coated strip was evaluated next. The irradiation was done using a roll-toroll transport system attached to one of the beam lines of the tandem Van de Graaff accelerator. For the roll-to-roll irradiation of the $46 \mathrm{~mm}$ wide tape, the ion beam was shaped into a rectangle approximately 5 by $1 \mathrm{~cm}$ with the long dimension oriented along the length of the tape. In order to uniformly irradiate the strip the ion beam was rastered at approximately over $70 \mathrm{~mm}$ at a frequency of $50 \mathrm{~Hz}$. The ion beam in the wide tape irradiations consisted of $\mathrm{Au}^{6+}$ ions with an energy of $16 \mathrm{MeV}$ and a particle current of $120 \mathrm{nA}$. In the first experiment the tape transport speed was varied every 3 meters to change the dose from $2 \times 10^{11} \mathrm{~A} / \mathrm{cm}^{2}$ to $10 \times 10^{11}$ $\mathrm{A} / \mathrm{cm}^{2}$ in order to identify the optimum dosage. Between each speed change, the ion beam was blocked to leave an unirradiated control section. After the irradiations of all the dosages were completed, the $46 \mathrm{~mm}$ strip was roll slit in to four $10 \mathrm{~mm}$ wide tapes.

Figure 4 shows that the $77 \mathrm{~K}$, self-field $I_{c}$ and $T_{c}$ both decrease as the dose of Au ions increases. At the previously determined optimal dose of $6 \times 10^{11} \mathrm{Au} / \mathrm{cm}^{2}$ the self-field $I_{c}$ decreases by about $30 \%$ and the $T_{c}$ falls by $2 \mathrm{~K}$. The $I_{c}$ enhancement as a function of dose was consistent with that measured with the static samples, with the optimal dose at $6 \times 10^{11} \mathrm{Au} / \mathrm{cm}^{2}$. The $I_{c}$ enhancement, measured across the width of the $46 \mathrm{~mm}$ tape, was consistent confirming the uniformity of the irradiation as seen in Fig. 5.

Using the optimal Au dose, an $80 \mathrm{~m}$ length of $46 \mathrm{~mm}$ wide production tape was irradiated in the roll-to-roll configuration. The tape speed was approximately $10 \mathrm{~m} / \mathrm{hr}$. After the irradiation, the $46 \mathrm{~mm}$ strip was roll slit into $4 \mathrm{~mm}$ 'insert wires' that were laminated with a $\mathrm{Cu}$ stabilizer forming a standard coil wire. The $77 \mathrm{~K}$ self-field transport $(1 \mathrm{~m}$ 
measurement length) $I_{c}$ of the unirradiated wire along with the short length $I_{c}$ of samples taken from the ends of the $46 \mathrm{~mm}$ wide strip before irradiation are shown in Fig. 6. The uniformity of $I_{c}$ and the consistent decrease from the unirradiated sample confirm the uniformity of the irradiation over the $80 \mathrm{~m}$ length.

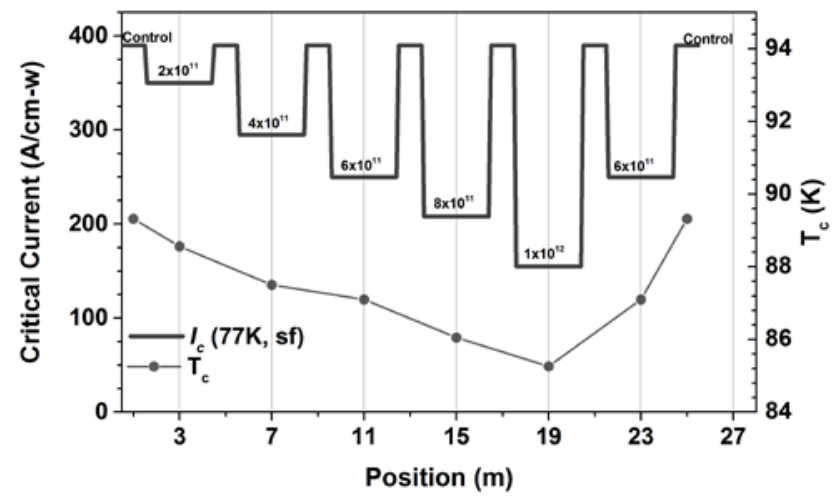

Fig. 4. $I_{c}$ and $T_{c}$ as a function of dose of $16 \mathrm{MeV}$ Au ions after irradiation of a moving $46 \mathrm{~mm}$ wide production coated conductor tape.

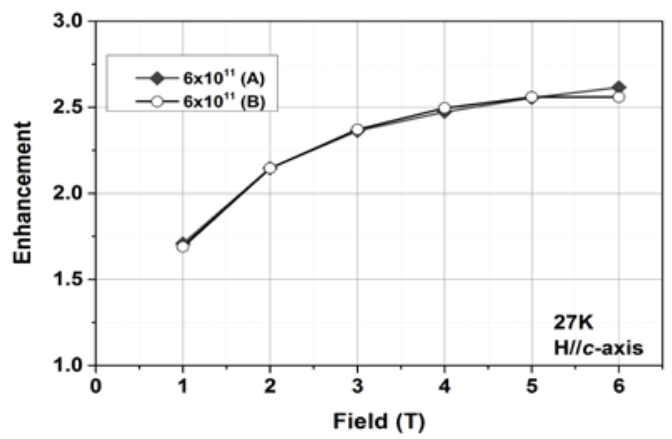

Fig. 5. $I_{c}$ enhancement measured in two separate $10 \mathrm{~mm}$ tapes slit from the $46 \mathrm{~mm}$ strip after the roll-to-roll irradiation.

Figure 7 shows the $I_{c}$ of short samples of the optimally irradiated wire as a function of field $(\mathrm{H} / / \mathrm{c})$ and temperature. The Au irradiation results in an enhancement of 2-fold or more at fields over $1 \mathrm{~T}$. For comparison, the $I_{c}$ of an unirradiated control sample at $30 \mathrm{~K}$ is included in the plot.

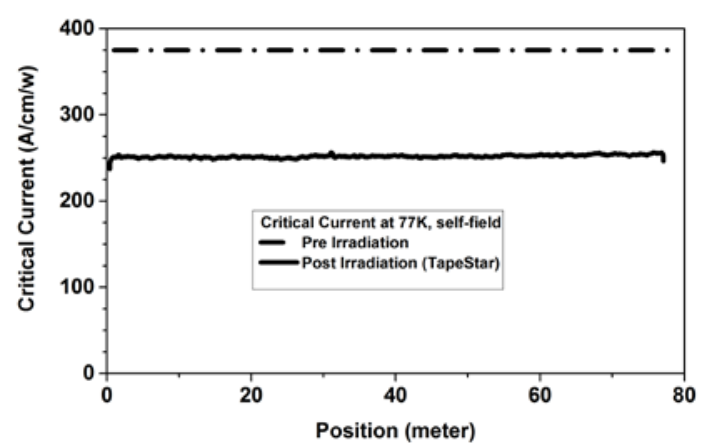

Fig. 6. $I_{c}(77 \mathrm{~K}$, self-field) as a function of length for a coil wire made from a $46 \mathrm{~mm}$ strip after irradiation to a dose of $6 \times 10^{11} \mathrm{Au} / \mathrm{cm}^{2}$. The preirradiation value is estimated from pieces cut from the ends of the original $46 \mathrm{~mm}$ strip.

\section{SUMMARY}

We demonstrated the feasibility of enhancing the pining in production length $2 \mathrm{G}$ wire using a roll-to-roll irradiation process. Irradiation of the $2 \mathrm{G}$ wire with a dose of $6 \times 10^{11} \mathrm{Au}$ ions $/ \mathrm{cm}^{2}$ results in a doubling of the critical current of AMSC's standard coil wire in the $4-50 \mathrm{~K}$ operating regime targeted for rotating machine applications and high field magnet applications. The roll-to-roll irradiation was carried out with ion energies under $20 \mathrm{MeV}$, which are readily accessible with commercial electrostatic generators. Further optimization of the ion species and energy promises to lead to additional improvements in $I_{c}$ and process rates required for incorporation into low-cost roll-to-roll manufacturing.

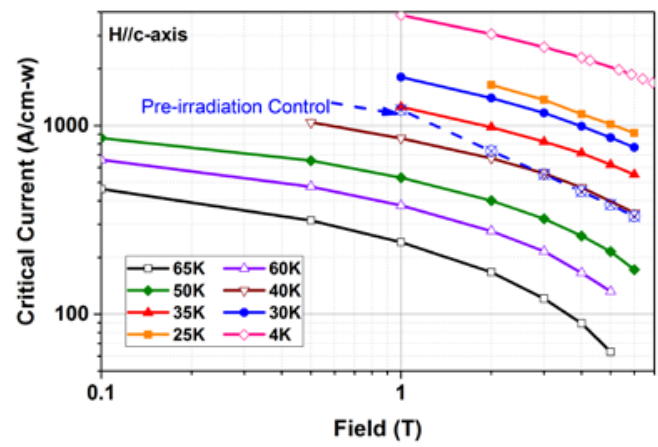

Fig. 7. $I_{c}$ as a function of temperature and field $(\mathrm{H} / / \mathrm{c})$ for a Au irradiated wire. The $I_{c}$ for an unirradiated control wire at $30 \mathrm{~K}$ (open circles with crosses) is included for comparison.

\section{REFERENCES}

[1] A.P. Malozemoff, "The power grid and the impact of high-temperature superconductor technology: An overview", in Superconductors in the Power Grid, C. Rey, ed. Elsevier, Amsterdam (2015) ch1.

[2] X. Obradors and T. Puig, "Coated conductors for power applications: materials challenges,” Supercond. Sci. Technol. 27, 044003 (2014).

[3] Y. Shiohara, T. Taneda, and M. Yoshizumi, "Overview of Materials and Power Applications of Coated Conductors Project,” Jpn. J. Appl. Phys. 51, 010007 (2012).

[4] V. Selvamanickam, M. H. Gharahcheshmeh, A. Xu, Y. Zhang and E. Galstyan, "Critical current density above $15 \mathrm{MA} \mathrm{cm}^{2}$ at $30 \mathrm{~K}, 3 \mathrm{~T}$ in 2.2 $\mu \mathrm{m}$ thick heavily-doped (Gd,Y)Ba2Cu3Ox superconductor tapes," Supercond Sci. Tech. 28, 072002 (2015).

[5] Y. Jia et al.,"Doubling the critical current density of high temperature superconductors through proton irradiation," Appl. Phys. Lett. 103, 122601 (2013).

[6] M. Leroux et al., "Rapid doubling of the critical current of $\mathrm{YBa}_{2} \mathrm{Cu}_{3} \mathrm{O}_{7-\delta}$ coated conductors for viable high-speed industrial processing," (submitted for publication)

[7] M.W. Rupich et al., "Second generation wire development at AMSC," IEEE Trans. Appl. Supercond. 23, 6601205 (2013).

[8] A. M. Campbell, and J. E. Evetts, "Flux vortices and transport currents in type II superconductors,” Adv. Phys. 21, 199 (1972).

[9] H. Matsui et.al., "Enhancement of critical current density in YBa2Cu3O7 films using a semiconductor ion implanter," J. Apl. Phys. 117, 043911 (2015).

[10] J. F. Ziegler, J. P. Biersack and, M. D. Ziegler, SRIM, The Stopping and Range of Ions in Matter.

[11] Y. Yeshurun, A. P. Malozemoff, and A. Shaulov, "Magnetic relaxation in high-temperature superconductors," Rev Mod. Phy. 68, 911 (1996).

[12] G. Blatter, M. V. Feigel'man, V. B. Geshkenbein, A. I. Larkin and V. M. Vinokur, "Vortices in high-temperature superconductors," Rev. Mod. Phy. 66, 1125 (1994).

[13] A. P. Malozemoff and M. P. A. Fisher, "Universality in the current decay and flux creep of Y-Ba-Cu-O high-temperature superconductors,"Phy. Rev. B, 426784 (1990).

[14] J. Thompson, et al, "Effect of flux creep on the temperature dependence of the current density in Y-Ba-Cu-O crystals," Phys. Rev. B 47, 14440 (1993).

[15] $\mathrm{H}$. Haberkorn et al., "High-temperature change of the creep rate in $\mathrm{YBa}_{2} \mathrm{Cu}_{3} \mathrm{O}_{7-\delta}$ films with different pinning landscapes," Phys. Rev. B 85, 174504 (2012). 